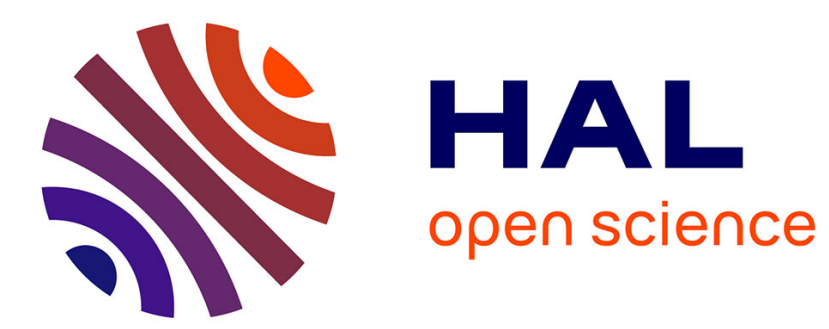

\title{
Interaction of Grains in Dusty Plasmas
}

A. Ignatov

\section{To cite this version:}

A. Ignatov. Interaction of Grains in Dusty Plasmas. Journal de Physique IV Proceedings, 1997, 07 (C4), pp.C5-215-C5-223. 10.1051/jp4:1997417 . jpa-00255573

\section{HAL Id: jpa-00255573 https://hal.science/jpa-00255573}

Submitted on 1 Jan 1997

HAL is a multi-disciplinary open access archive for the deposit and dissemination of scientific research documents, whether they are published or not. The documents may come from teaching and research institutions in France or abroad, or from public or private research centers.
L'archive ouverte pluridisciplinaire HAL, est destinée au dépôt et à la diffusion de documents scientifiques de niveau recherche, publiés ou non, émanant des établissements d'enseignement et de recherche français ou étrangers, des laboratoires publics ou privés. 


\title{
Interaction of Grains in Dusty Plasmas
}

\author{
$\underline{\text { A.M. Ignatov }}$
}

General Physics Institute, 38 Vavilova Str., Moscow 117942, Russia

\begin{abstract}
Plasma absorption by dust grains results in anisotropy of plasma distribution function. This yields the effective attractive interaction between grains. Here a brief review of physical properties of this interaction is given. First we demonstrate that there is the attracting force between two grains obeying the inverse square low. Then a set of kinetic equations taking into account both attracting and repulsing forces is proposed. We study a simple model of a dust cloud consisting of finite number of grains. It is shown that even within gaseous approximation a dust cloud is a compact object with a sharp boundary. Finally, we discuss recent experiments aimed to detecting the attractive force between massive bodies
\end{abstract}

\section{Introduction}

Both space and laboratory low-temperature plasmas are often polluted with a certain number of dust grains. The size of these grains varies over a very wide range from nanometers to micrometers. Since a dust grain can carry additional electric charge, sometimes as high as $10^{4}$ elementary charges, whose amount depends on the state of surrounding plasma, relatively low dust concentration can crucially change the plasma properties. This medium composed of several species of particles with fixed charge (electrons and ions) and variable charge (dust grains), which is referred to as a dusty plasma, exhibits many specific properties.

During the recent decade a great number of studies on dusty plasmas were performed. The review of these studies may be found, e.g., in Refs. [1,2]. Also, many original works on dusty plasma were presented at the ICPIG meetings: In the present paper, we focus on the following specific problem. If the dust grain is relatively large (more than several micrometers under typical laboratory conditions), it behaves like a floating Lengmuir probe. A grain absorbs plasma particles hitting its surface and tunes its charge and electric potential in such a way that the net electric current is zero. As a result, the net charge of an individual grain is always negative. However, the dust often forms various patterns, like crystals, clusters or clouds. Obviously, to maintain the stability of the ensemble of likely charged particles some additional, either external or attracting intrinsic forces are required.

Although under laboratory conditions external forces like the external electrostatic field or the gravitational field of the Earth are extremly important, there is question of general interest: If there are any attracting forces between likely charged particles in a medium? Generally, there are numerous examples of the so-called overscreening, but in application to dusty plasma a first attempt to answer this question, to my knowledge, was made only three years ago by Tsytovich [3]. He showed that the electrostatic interaction energy of two grains decreases as the distance between them decreases to the values less than the Debye length, and this is a hint at the electrostatic attraction of some kind. Another possibility dicussed in the literature is that the static dielectric permittivity in nonequilibrium plasmas often changes its sign, that results in the oscillatory interparticle potential. 
For example, this may arise due to the ion flow past the dust grains [4,5]. Of interest also is the nonlinear Debye screening discussed in application to colloidal plasmas [6]. This process resembles the formation of the hydrogen bond in electrolytes: two particulates (macroions) each carrying sufficiently large negative charge can form a stable complex collecting a clond of positive charges between them.

All mentioned processes can provide the attraction of grains at relatively small distance, of the order of the Debye length. In the present paper we focus on another mechanism giving rise to the attraction at much larger distances $[7,8]$. It originates from a mechanistic theory of the universal gravitation proposed in the 18-th century by G.L. Lesage and may be outlined as follows [9]. He was an adept of the ancient atomistics and it was evident for him that the ether consists of tiny atoms moving in random directions. Lesage's main hypothesis was that an ether atom hitting a massive body loses a small part of its vital force. If there are two bodies, then the ether flux coming through the first body is smaller in the direction of the second body, hence, the effective attracting force must arise. The reduction of the ether flux and, consequently, the force acting upon the first body are proportional to the solid angle, at which the second body is visible, and the latter is proportional to the inverse distance squared.

Although this ingenuous theory has many obvious drawbacks and, to tell the truth, even two hundred years ago very few accepted it seriously, it is evident that a similar process may be relevant in a plasma. Since the interaction of plasma particles with the solid surface is practically always inelastic, we can apply Lesage's theory to a plasma merely replacing in his reasoning the words "vital force" by the more habitual "momentum". Moreover, a plasma may be even a more favourable medium for providing the attraction of this kind because a massive body often absorbs plasma particles. In other words, a massive body in a plasma, e.g., a floating probe, creates a converging plasma flow, and evidently there should arise some drag force acting upon any other object in its vicinity $[7,8]$.

The existence of this force was confirmed in computer experiments by Khodataev et al. [10]. Recently two attempts were made to measure the force between massive bodies in a plasma which we briefly describe below.

This paper is organised as follows. In section 2 the essence of the Lesage gravity is illustrated using a simple model of two grains with absorbing surface. Then we formulate the kinetic equations for dusty plasma taking into account absorption of plasma by dust grains (Sec. 3). In section 4 a simple model of a dust cloud sustained by the Lasage gravity is discussed. We briefly describe the preliminary results of two known experiments aimed to detect this interaction in Sec. 5 .

\section{Attraction of two dust grains}

\section{$2.1 \quad$ Large grains}

We start with the case of relatively large dust grains with radiuses, $a_{i}$, exceeding the Debye length, $\lambda_{D}$, of the surrounding plasma. Let us first consider the dynamics of ions. Since the electric potential is nonzero in the thin layer around the grain only, we ignore the influence of the electric field in the bulk of the plasma. So it is natural to assume that the only way the grain influences the ionic distribution is the absorption of ions hitting its surface.

The trajectories of plasma particles deviate from straight lines at the nearest vicinity of dust grains, that is, the ionic distribution function is zero if a straight line coming from infinity to the observation point $\mathbf{r}$ intersects one of the grains. With a set of spherical dust grains placed at $\mathbf{r}=\mathbf{R}_{\mathbf{i}}$, this distribution may be written as

$$
f^{(i)}(\mathbf{r}, \mathbf{v})=f_{0}^{(\mathbf{i})}(\mathbf{v}) \prod_{\mathbf{i}} \mathbf{g}\left(\mathbf{v}, \frac{\mathbf{r}-\mathbf{R}_{\mathbf{i}}}{\mathbf{a}_{\mathbf{i}}}\right),
$$


where $g(\mathbf{v}, \mathbf{x})=\theta\left(\mathbf{v} \sqrt{\mathbf{x}^{2}-\mathbf{1}}-\mathbf{v} \mathbf{x}\right)$ and $f_{0}^{(i)}(\mathbf{v})$ is the distribution function at infinity. This distribution function is zero inside a certain number of cones in the velocity space and it is equal to the unperturbed distribution otherwise.

The force upon a grain is the momentum flux through its surface. It was evaluated using the distribution (1) and the force between two grains was found to be [8]

$$
F=\frac{3 \pi}{4} n_{0}^{(i)} T_{i} \frac{a_{1}^{2} a_{2}^{2}}{R^{2}}
$$

where $n_{0}^{(i)}$ is the density, $T_{i}$ is the ion temperature and $R$ is the distance between the centres of the grains. Since in performing these calculations we have just improved the Lesage's reasoning in a little more advanced fashion, in what follows this mechanism of attraction is called the Lesage gravity. Of interest is that this model reproduces the well-known feature of the Newtonian gravitation: two spherical bodies interact as point masses.

The force (2) is independent of the ion mass, and the same expression, at first sight, should be valid for electrons also. However, in assuming the total absorption, the current density of electrons and ions entering the grain within this model is by the order of magnitude the random current, $n_{0}^{\alpha} \sqrt{T_{\alpha} / m_{\alpha}} \quad(\alpha=e, i)$, while in equilibrium the electron current must be equal to the ion current. This means that only a small part of electrons $\left(\sqrt{m_{e} / m_{i}}\right.$ if $\left.T_{e} \approx T_{i}\right)$ is actually absorbed, and the remaining part is scattered by the Debye shield. Since $a \gg \lambda_{D}$, the scattering results in mirror reflection for the bulk of electrons.

Evidently, for the Lasage gravity to exist some energy absorption in particle-dust collisions is required. The mirror reflection gives no rise to the anisotropy and the electron distribution function remains nearly Maxwellian. Thus the electron component of the attracting force is at least $\sqrt{m_{e} / m_{i}}$ times less than the ion force (2) and, therefore, is negligible.

It is of importance that, unlike the genuine gravitation, the Lesage gravity is a non-pairwise interaction. Moreover, with other types of boundary conditions at the grains, Newton's third low may no longer hold. Generally, such concepts as a field or a potential are inapplicable to this force $[8]$.

\subsection{Small grains}

Let us estimate now how the Lesage gravity is modified if the grains' radiuses are much smaller than the Debye length. Suppose there is a single dust grain with the radius $a_{1}$. Let us denote the ion current entering the grain as $e J_{i}$; by the order of magnitude $J_{i}=a_{1}^{2} n_{0}^{(i)} \sqrt{T_{i} / m_{i}}$. Due to the conservation of the number of ions in a plasma, there is a constant flow converging to the grain with the velocity

$$
v_{r}(r)=-\frac{1}{4 \pi r^{2}} \frac{J_{i}}{n^{(i)}(r)} .
$$

At the distance exceeding few Debye lengths we can neglect the deviation of ion density from its equilibrium value, $n^{(i)}(r) \approx n_{0}^{(i)}$. It should be noted that since Eq. (3) is a solution of the continuity equation, it is insensitive to all types of collisions conserving the number of particles.

Suppose that there is some object, say, another grain with radius $a_{2}$. Since the second grain is immersed into the plasma flow, there should be the drag force acting upon it. Evidently, the direction of the drag corresponds to the effective attraction of the grains, and if $v_{r}(r)$ is small compared to the ion thermal velocity, the force has to be proportional to $v_{r}(r)$.

The ion drag force acting upon a dust grain was evaluated in [12]. Generally, there are two processes: the drag provided by ions collected by the grain, which is an analogue of the discussed above Lesage force, and the momentum transfer by ions scattered in the electric field of the grain, which was discarded in the previous discussion. (Again, the electron drag force is negligible.) If 
$a \ll \lambda_{D}$, then both these forces are of the same order of magnitude and the total force may be estimated as $F \approx a_{2}^{2} \sqrt{T_{i} m_{i}} n_{i} v_{r}(r)$. Combining the latter with Eq.(3) results in

$$
F \approx n_{0}^{(i)} T_{i} \frac{a_{1}^{2} a_{2}^{2}}{R^{2}}
$$

Comparing this with Eq.(2) we conclude that the dependence of the attracting force on the ratio $a / \lambda_{D}$ is rather weak and is introduced by a coefficient of order unity.

\section{$3 \quad$ Kinetic equations}

Due to the mentioned peculiarities of the Lasage gravity we cannot use a pairwise potential of any kind to describe the interaction of grains in a large ensemble. The only way to take into account this attracting force is simultaneous solution of kinetic equations for plasma and dust. Unfortunately, the problem of consistent derivation of kinetic equations for a dusty plasma, to my knowledge, is far from satisfactory solution, and we have no other choice but to guess these equations. Here the following set of kinetic equations describing a large number of dust grains immersed in a plasma is used [11]:

$$
\begin{array}{r}
\frac{\partial f_{\alpha}(t, \mathbf{r}, \mathbf{p})}{\partial t}+\frac{\mathbf{p}}{m_{\alpha}} \frac{\partial f_{\alpha}(t, \mathbf{r}, \mathbf{p})}{\partial \mathbf{r}}+e_{\alpha} \mathbf{E}(\mathbf{r}) \frac{\partial f_{\alpha}(t, \mathbf{r}, \mathbf{p})}{\partial \mathbf{p}}=I_{\alpha}^{(a)}+I_{\alpha}^{(c)} \\
\frac{\partial f_{d}(t, \mathbf{r}, \mathbf{p}, Q)}{\partial t}+\frac{\mathbf{p}}{m_{d}} \frac{\partial f_{d}(t, \mathbf{r}, \mathbf{p}, Q)}{\partial \mathbf{r}}-Q \mathbf{E} \frac{\partial f_{d}(t, \mathbf{r}, \mathbf{p}, Q)}{\partial \mathbf{p}}=I_{d}^{(\alpha)}+I_{d}^{(c)} \\
\nabla \mathbf{E}=4 \pi \int d \mathbf{p}\left(\sum_{\alpha} e_{\alpha} f_{\alpha}(\mathbf{r}, \mathbf{p})-\int d Q Q f_{d}(\mathbf{r}, \mathbf{p}, Q)\right)
\end{array}
$$

Electron and ion variables here are distinguished with the lower index $\alpha=e, i$, the charge of the dust is $-Q<0$, other notation is standard. Since the charge of each grain is determined by the state of the surrounding plasma, in using the kinetic approach it should be treated as an additional intrinsic variable of the dust component, i.e., the dust distribution function, $f_{d}$, besides the coordinate and the momentum depends on the grain charge, $Q$.

Generally, there are many various collisional processes in a dusty plasma. For simplicity only two of them are taken into account: (i) inelastic collisions of plasma particles with dust grains resulting in absorption of electrons and ions, and (ii) elastic scattering of plasma particles in the electric field of a grain. The collision terms in the right-hand sides of Eqs. $(5,6)$ are obtained considering the probabilities of corresponding processes [11]. Assuming that all dust grains are of the same size, $a$, small compared to the Debye length and $m_{\alpha}<<m_{d}$, the terms corresponding to the absorption of a plasma by dust grains look like:

$$
\begin{array}{r}
I_{\alpha}^{(a)}(\mathbf{r}, \mathbf{p})=-\int d Q v_{\alpha} \sigma\left(v_{\alpha}, Q\right) n_{d}(\mathbf{r}, Q) f_{\alpha}(\mathbf{r}, \mathbf{p}) ; \quad \alpha=e, i \\
I_{d}^{(a)}(\mathbf{r}, \mathbf{p}, Q)=-\frac{\partial}{\partial \mathbf{p}}\left(\mathbf{A}^{(\alpha)}(\mathbf{r}) f_{d}(\mathbf{r}, \mathbf{p}, Q)\right)-\frac{\partial}{\partial Q}\left(J(\mathbf{r}, Q) f_{d}(\mathbf{r}, \mathbf{p}, Q)\right)
\end{array}
$$

where $v_{\alpha}=p / m_{\alpha}$

$$
\begin{array}{r}
n_{d}(\mathbf{r}, Q)=\int d \mathbf{p} f_{d}(\mathbf{r}, \mathbf{p}, Q) \\
J(\mathbf{r}, Q)=\sum_{\alpha=e, i} e_{\alpha} \int d \mathbf{p} v_{\alpha} \sigma_{\alpha}\left(v_{\alpha}, Q\right) f_{\alpha}(\mathbf{r}, \mathbf{p}) \\
\mathbf{A}^{(a)}(\mathbf{r})=\sum_{\alpha=e, i} \int d \mathbf{p} \mathbf{p} v_{\alpha} \sigma_{\alpha}\left(v_{\alpha}, Q\right) f_{\alpha}(\mathbf{r}, \mathbf{p}) .
\end{array}
$$


The physical meaning of these quantities is evident: $J(\mathbf{r}, Q)$ is the net electric current at a dust grain with the charge $Q$ situated at the point $\mathbf{r}$, while $\mathbf{A}^{(\mathbf{a})}(\mathbf{r})$ is the drag force due to plasma absorption acting upon it, that is, the Lesage force. The absorption cross-section, $\sigma_{\alpha}$, is usually obtained assuming that a grain traps all particles coming at the distance smaller than its radius from the point charge $-Q$ with the Coulomb potential:

$$
\sigma_{\alpha}(v, Q)=\pi a^{2} \theta\left(1+\frac{2 Q e_{\alpha}}{a m_{\alpha} v^{2}}\right)
$$

Electrons are repulsed from a grain $\left(e_{e}<0\right)$ and this. cross-section is nonzero for rather large $v$, normally exceeding several thermal velocities. As for ions, the expression (11) is divergent for small values of velocity, that is, all slow ions are collected by a single grain. To avoid this evidently non-physical divergence some kind of cut-off is needed, which is discussed below.

Coulomb scattering is introduced by collision terms in Eqs. $(5,6)$ marked with the upper index (c). As a simplest approximation we use Landau's collision integral resulting in additional Coulomb drag force

$$
\dot{I}_{d}^{(c)}(\mathbf{r}, \mathbf{p}, Q)=-\frac{\partial}{\partial \mathbf{p}}\left(\mathbf{A}^{(\mathrm{c})}(\mathbf{r}) f_{d}(\mathbf{r}, \mathbf{p}, Q)\right)
$$

where

$$
\mathbf{A}^{(c)}(\mathbf{r})=\sum_{\alpha} 4 \pi e^{2} Q^{2} L m_{\alpha} \int d \mathbf{p} \frac{\mathbf{p}}{p^{3}} f_{\alpha}(\mathbf{r}, \mathbf{p})
$$

and $L=\ln \left(\lambda_{D} / a\right)$. Coulombian collision term for the plasma component in Eq. (5) is given by the Landau integral.

Kinetic equations $(5,6)$ combined with collision terms $(8,9,12)$ and the Poisson equation (7) form a complete set. This set guarantees the conservation of the total charge and momentum of the plasma-dust system, but the energy and the number of plasma particles are no longer conserved. It is evident that to describe a steady state of a dusty plasma within this model one should take into account external plasma sources of some kind. In what follows we consider a compact dust pattern consisting of finite number of grains and assume that these sources are removed to infinity where the plasma distribution function is Maxwellian.

It should be noted the kinetic equations written above obviously describe nonstationary grain charging. The equation for the average grain charge is easily obtained integrating Eq. (6) over $\mathbf{p}$ and $Q$. However, it generally differs from that proposed in [13] where the dispersion over the grain charge was neglected.

\section{Dust cloud}

\subsection{Basic equations}

In this section we discuss the simplest model of a rarefied compact dust cloud. Let us consider an ensemble of $N(N \gg 1)$ immobile dust grains randomly distributed in space. Assuming that the plasma is steady, i.e., all time derivatives are zero, the first two momenta of Eq. (6) are written as

$$
\begin{array}{r}
J(\mathbf{r}, Q) n_{d}(\mathbf{r}, Q)=0, \\
(Q \mathbf{E}(\mathbf{r})-\mathbf{A}(\mathbf{r})) n_{d}(\mathbf{r}, Q)=0,
\end{array}
$$

where $\mathbf{A}=\mathbf{A}^{(a)}+\mathbf{A}^{(c)}$ is the total drag force acting upon the dust component. Eq. (14) guarantees the absence of the net electric current in the equilibrium state. Its only solution is $n_{d}(\mathbf{r}, Q)=$ $n_{d}(\mathbf{r}) \delta\left(Q-Q_{0}(\mathbf{r})\right)$, where $Q_{0}(\mathbf{r})$ is the self-consistent charge of the dust component obeying the 
relation $J\left(\mathbf{r}, Q_{0}(\mathbf{r})\right)=0$. The value of the grain charge is estimated as $Q \approx a T_{e} / e$. It is determined mainly by the electron temperature and its dependence on other plasma parameters is fairly weak. In what follows we ignore the spatial variation of the grain charge.

The structure of the dust cloud is described by the equation of the momentum balance (15). Evidently, in the absence of external forces the cloud is spherically symmetric. To evaluate the Lesage force we have to solve the kinetic equations (5) with some dust distribution $n_{d}(r)$. This solution was obtained in powers of the dust density and the linear term of expansion was found to be [11]

$$
f_{\alpha}(\mathbf{p}, \mathbf{r})=f_{0 \alpha}(p)\left(1-\sigma_{\alpha}\left(v_{\alpha}\right) \lambda\left(r, \mathbf{v}_{\alpha}\right)-\frac{e_{\alpha}}{T_{\alpha}} \phi(r)\right)
$$

where $\mathbf{E}=-\nabla \phi$,

$$
\lambda(r, \mathbf{v})=\int_{R_{0}}^{\infty} \frac{r^{\prime} d r^{\prime}}{\sqrt{r^{\prime 2}-R_{0}^{2}}} n_{d}\left(r^{\prime}\right)+\operatorname{sign}\left(v_{r}\right) \int_{R_{0}}^{r} \frac{r^{\prime} d r^{\prime}}{\sqrt{r^{\prime 2}-R_{0}^{2}}} n_{d}\left(r^{\prime}\right)
$$

and $R_{0}=|\mathbf{r} \times \mathbf{v}| / v$. The deviation of the distribution function (16) from its equilibrium value is provided, first, by the electric potential, $\phi(r)$, and second, by the plasma absorption. The latter contribution is introduced by the non-local dependence on the dust density because the probability of absorbing a plasma particle depends on a path it reaches the observation point.

Straightforward integration of Eq. (16) results in expressions for the plasma density and the Lesage force acting upon the dust component. The density deviation is caused by the self-consistent electric potential and the plasma absorption

$$
n_{\alpha}(r)=n_{0}\left(1-\frac{e_{\alpha}}{T_{\alpha}} \phi(r)-\kappa_{\alpha}(r)\right)
$$

where

$$
\kappa_{\alpha}(r)=\frac{1}{2 n_{0}} \int_{0}^{\infty} v^{2} d v \sigma_{\alpha}(v) f_{0 \alpha}(v) \int_{0}^{\infty} \frac{r^{\prime} d r^{\prime}}{r} \ln \left|\frac{r+r^{\prime}}{r-r^{\prime}}\right| n_{d}\left(r^{\prime}\right)
$$

The drag force, $\mathbf{A}$, is independent of the electric field and determined by the dust density only:

$$
A_{r}=-\beta \frac{1}{r^{2}} \int_{0}^{r} r^{2} d r^{\prime} n_{d}\left(r^{\prime}\right)
$$

where

$$
\beta=4 \pi \sum_{\alpha} \int_{0}^{\infty} d v f_{0 \alpha}(v)\left\{m_{\alpha} v^{4} \sigma_{\alpha}(v)^{2}+\frac{4 \pi e^{2} Q^{2} L}{m_{\alpha}} \sigma_{\alpha}(v)\right\} .
$$

Regardless the above mentioned singularity of the absorption cross-section (11), the integrals for the plasma density (19) and for the net electric current (10) are convergent. At the same time, the second term in the integrand of Eq. (21) diverges for small $v$. This divergence is provided by multiplication of two factors. On one hand, dust absorbs mostly slow ions, and, on the other hand, the Coulombian drag force given by Eq. (13) is very sensitive to the details of the distribution in a range of small ion velocities. (It is evident that the influence of the electrons is negligible.) Of importance is that the integrand in (21) behaves like $1 / v^{2}$, that is, introducing any kind of cut-off one can hope for qualitative result only.

There are two ways to get rid of this divergence. First, one should take into account the nonlinear corrections to the distribution function (16). It is a complicated task which I managed to solve in the absence of the electric field only [12]. In this case the ionic distribution roughly behaves as 
$\exp \left(-v_{1}^{2} / v^{2}\right)$, where $v_{1} \approx v_{T_{i}} a N^{1 / 2} / R_{0}$ with $R_{0}$ being the characteristic size of the dust cloud. Second, the cross-section (11) presumably cannot exceed $\pi \lambda_{D}^{2}$ that results in another cut-off velocity $v_{2} \approx v_{T_{i}} a / \lambda_{D}$. In the following estimations the second cut-off velocity is used - this mechanism prevails if the total number of grains is not too large, namely, $N \ll R_{\tilde{\sigma}}^{2} / \lambda_{D}^{2}$. In this case the integral (21) is estimated as $\beta \approx n_{0} a^{3} \lambda_{D} T_{i}$.

\subsection{Force balance}

The steady state of a dust cloud is described by the condition of the momentum balance (15) with the drag force given by (20) combined with the Poisson equation

$$
\Delta \phi-\frac{1}{r_{D}^{2}} \phi=4 \pi\left(Q n_{d}(r)-n_{0} \sum_{\alpha} e_{\alpha} \kappa_{\alpha}(r)\right) .
$$

Consider first the force balance far from the cloud. Since the total number of particles is finite, the dust density should decrease at large distances, $n_{d}(r)<1 / r^{3}$. This means that the drag force (20) behaves like $A_{r} \approx \beta N / 4 \pi r^{2}$. As it follows from the integral (19), $\kappa_{\alpha}(r) \propto 1 / r^{2}$ at $r \rightarrow \infty$, that is the potential $\phi(r) \propto 1 / r^{2}$ and the electric field $E_{r} \propto 1 / r^{3}$. Therefore, the force attracting grains at large distance always exceeds the repulsing electric force.

In this situation, the only way to satisfy the momentum balance equation (15) is to put $n_{d}(r)=0$. However, the dust density is not identically zero, that is, there should exist some limiting radius, $R_{0}$, such that $n_{d}(r)=0, Q E_{r}<A_{r}$ for $r>R_{0}$, and $n_{d}(r) \neq 0, Q E_{r}=A_{r}$ for $r<R_{0}$. In other words, the plasma-dust cloud is not a diffuse object with smooth density profile, but it is a compact formation with a sharp boundary. It should be stressed that the asymptotic behaviour of attracting and repulsing forces is of geometrical origin, that is, this conclusion is independent of the problems with evaluating the drag coefficient (21).

To determine the radius of the dust cloud let us consider the force balance inside it. Assuming the $R_{0} \gg \lambda_{D}$, we implement Eq. (20) and the plasma neutrality condition $\sum_{\alpha} e_{\alpha} n_{\alpha}-Q n_{d}=0$ to rewrite the force balance equation as

$$
\frac{1}{r^{2}} \frac{d}{d r} r^{2} \frac{d}{d r}\left(n_{d}(r)+\frac{n_{0}}{Q} \sum_{\alpha} e_{\alpha} \kappa_{\alpha}(r)\right)=-\frac{n_{d}(r)}{b^{2}},
$$

where

$$
b^{2}=\frac{4 \pi Q^{2} \lambda_{D}^{2}}{\beta}
$$

fixes the spatial scale of the cloud, that is, $R_{0} \propto b$. According to the estimations written above, $b^{2} \approx \lambda_{D}^{3} / a$. The second term in the left-hand side of Eq. (23) arises due to the absorption of plasma. It is estimated as $n_{d} a / \lambda_{D}$, therefore, evaluating the electric field inside the cloud we can neglect the absorption. Eq. (23) is now easily solved resulting in

$$
n_{d}(r)=C \frac{\sin (r / b)}{r} ; \quad r<R_{0}
$$

where $C$ is a normalising coefficient. Since the expression (25) changes its sign as $r$ exceeds $\pi b$, the cloud radius must be smaller than $\pi b$. On the other hand, any stepwise discontinuity of dust density would result in high electric field at the edge of the cloud, and it is impossible to counterbalance the strong repulsing force by the attracting drag force. Thus, the only way to maintain the force balance is to put $R_{0}=\pi b$. In this case, the dust density at the edge of the cloud is zero, and no high electric field arises. The normalising coefficient, $C$, is now easily expressed in terms of the number of dust grains:

$$
C=\frac{N}{4 \pi^{2} b^{2}}
$$


The range of validity of the expansion in powers of the dust density may now be written as $N \ll \lambda_{D} / a$. With larger number of dust grains, the absorption of slow ions becomes significant.

It should be noted that although all equations we have solved are linear, the problem in general is nonlinear - the condition of force balance (15) is quadratic in $n_{d}$. In a sence, the obtained solution may be regarded as a standing shock wave bounding a dust cloud.

\section{$5 \quad$ Experiments}

The Lesage force should act between any massive bodies immersed in a plasma. Although one can hardly perform direct measurement of the forces between small dust grains, recently two attempts were made to detect the force between larger objects immersed in a plasma.

Sergeichev and Sychov [15] used two pieces of optical fiber $300 \mu \mathrm{m}$ in diameter and about $20 \mathrm{~cm}$ long fixed horizontally in the rf discharge. They observed some attraction of these strings with a microscope, but since it was very small, less than the diameter, no detailed measurements were performed.

Another attempt was made by the group from Arzamas-16 [11]. They have studied the attraction of massive bodies in the glow discharge in air $(0.01-0.1$ Torr $)$. First, two glass threads about $100 \mu \mathrm{m}$ in diameter and $45 \mathrm{~cm}$ long were freely hung along the direction of the discharge current. The distance between threads was $6 \mathrm{~mm}$. Obseved with an unaided eye, the approach of their ends was roughly proportional to the discharge current. However, when the direction of the discharge current was perpendicular to the threads no approach was observed. It was supposed that in this situation the attraction is provided by the Ampere force in the current-carrying plasma.

In the second series of experiments they used two thin $(10 \mu \mathrm{m})$ mylar ribbons $(8 \times 100 \mathrm{~mm})$ $5 \mathrm{~mm}$ distant from each other hanging down across the discharge current. With the growing current, the attraction between ribbons increased, so they could even clap. Seemingly, with this geometry of the experiment, the Ampere force is excluded, while due to the difference in surface areas of ribbons and threads all surface effects should be strongly enhanced. That is why one can assume that the attraction between ribbons is provided by the discussed above Lesage force.

\section{Acknowledgments}

I am grateful to Yu. Klimontovich, A. Rukhadze and S. Trigger for many helpful remarks, and to

K. Sergeichev and I. Sychev for discussions of their unfinished experiments.

This work has been supported by the Russian Foundation for Basic Research, projects \# 93-0203357, \# 96-02-16456.

\section{References}

[1] Dusty and Dirty Plasmas, Noise and Chaos in Space and in the Laboratory, Kikuchi H., Ed., N.-Y.: Plenum, 1994

[2] Tsytovich V.N., Uspekhi fiz. nauk,167, 57 (1997).

[3] Tsytovich V.N., Comm. Plasma Phys.,15, 349 (1994).

[4] Vladimirov S.V. Nambu M., Phys.Rev. E, 52, R2172 (1995)

[5] Melanso F. Goree J., J. Vac. Sci. Technol. A.,14, 511(1996).

[6] Allakhyarov E.A., Podlubny L.I., Schram P., Trigger S.A. Physica A., 220, 349(1995)

[7] Ignatov A.M., Bull. Lebedev Physics Inst. \# 1-2, 58 (1995). 
[8] Ignatov A.M., Fizika plasmy (Plasma Physics Reports), 22, 648 (1996).

[9] Rosenberger F., Die Geschichte der Physik, Dritter Teil, Braunschweig (1887)

[10] Khodataev Ya., Bingham R., Tarakanov V., Tsytovich V., Fizika plasmy (Plasma Physics Reports) 22, 1028 (1996).

[11] Ignatov A.M., Fizika plasmy (Plasma Physics Reports), to be published.

[12] Barnes M.S., Keller J.H., O'Neil J.A., Coultas D.H., Phys. Rev. Lett., 68, 313 (1992).

[13] Sitenko A.G., Zagorodny A.G., Chutov Yu.I., Schram P., Tsytovich V.N., Plasma Phys. Control. Fusion, 38, A105 (1996).

[14] Sergeichev K.F., Sychev I.A. , private communication.

[15] Dubinov A.E., Zhdanov V.S., Ignatov A.M., Kornilov S.Yu., Sadovoi S.A., Selemir V.D., Bull. Lebedev Physics Inst. \# 7-8 ( 1997), to be published 